\title{
Étude du comportement analytique de la mirtazapine en chromatographie liquide haute performance et électrophorèse capillaire de zone
}

\section{Analytical behaviour of mirtazapine in high performance liquid chromatography and capillary zone electrophoresis}

Laurence LABAT $^{*(1)}$, Philippe DALLET ${ }^{(1)}$, Marc DEVEAUX ${ }^{(2)}$, Jean-Pierre DUBOST ${ }^{(1)}$,

(1) Laboratoire de Chimie Analytique, UFR de Pharmacie, 3, place de la Victoire - 33076 BORDEAUX Cedex (2) Institut de Médecine Légale, Place Théo Varlet - 59000 LILLE

* Auteur à qui adresser la correspondance : Laurence LABAT, Laboratoire de Chimie Analytique,

UFR de Sciences Pharmaceutiques, 3, place de la Victoire - 33076 BORDEAUX Cedex Tél : 0557571818 - Fax : 0556947090 - E-mail : laurence.labat@chimana.u-bordeaux2.fr

(Reçu le 9 mai 2001 ; accepté le 29 mai 2001)

\section{$R \hat{E} S U M E ́$}

La mirtazapine est un antidépresseur de nouvelle génération, antagoniste des récepteurs $\alpha_{2}$ présynaptiques d'action centrale, permettant l'augmentation de la neurotransmission noradrénergique et sérotoninergique. L'identification et le dosage dans les milieux biologiques de cette molécule et de son métabolite actif, la déméthylmirtazapine, peuvent être réalisés par chromatographie liquide haute performance (CLHP) et par électrophorèse capillaire de zone (ECZ).

La mirtazapine possède deux fonctions amine de propriétés acido-basiques très différentes. Le $p K a$ du groupement amino-2-pyridine est de 3,60 $\pm 0,05$, et celui de la fonction amine tertiaire, de 7,50 $\pm 0,05$. Nous avons étudié l'incidence de ces constantes d'ionisation sur le comportement analytique de la mirtazapine parmi les antidépresseurs de nouvelle génération, dans le cadre du développement de méthodes de séparation en CLHP et en ECZ.

Nous présentons la séparation d'un mélange de 12 antidépresseurs, dont la mirtazapine et la déméthylmirtazapine, en CLHP sur une colonne Satisfaction $\AA R P 18$ AB maintenue à

\section{SUMMARY}

Mirtazapine is a new antidepressant, antagonist of central $\alpha_{2}$ presynaptic receptors, and thus increases the release of noradrenaline and serotonin. Determination in biological fluids of mirtazapine and demethylmirtazapine, the pharmacologically active metabolite, can be performed by high performance liquid chromatography (HPLC) and capillary zone electrophoresis (CZE).

Mirtazapine has two amino groups with different acido-basic properties. The $p K a$ of the amino-2-pyridine group was found to be $3.60 \pm 0.05$ and the pKa of the tertiary amine group, $7.50 \pm 0.05$. We studied the influence of these acidobasic characteristics on the analytical behaviour of mirtazapine among other new antidepressants, in developing HPLC and CZE separative technics.

We performed the separation of twelve antidepressants, including mirtazapine and demethylmirtazapine, by HPLC on a Satisfaction ${ }^{\circledR} R P 18$ AB column at $45^{\circ} \mathrm{C}$ with a mobile phase composition of acetonitrile/phosphate buffer $\mathrm{pH} 4.8$ $(35: 65, v / v)$. In CZE, we optimized the separation at $15^{\circ} \mathrm{C}$ 
$45^{\circ} \mathrm{C}$ avec une phase mobile acétonitrile/tampon $\mathrm{pH} 4,8$ (35: $65, v / v)$. En ECZ, la séparation a été optimisée à $15^{\circ} \mathrm{C}$ sur un capillaire en silice fondue conditionné par un tampon borate $42 m M(p H 9,5)$ et méthanol $(80: 20, v / v)$ et une tension appliquée de $15 \mathrm{kV}$.

En CLHP, nous avons mis en évidence l'influence particulièrement importante du $\mathrm{pH}$ de la phase mobile ( $\mathrm{pH} 3$ à $p H$ $4,95)$ et de la température $\left(25^{\circ} \mathrm{C}\right.$ à $\left.50^{\circ} \mathrm{C}\right)$ sur les temps de rétention de la mirtazapine et de son métabolite. En ECZ, le pH du tampon de migration (entre $p H$ 9,2 et $p H$ 9,9) a une influence homogène sur la migration de l'ensemble des antidépresseurs, tous faiblement ionisés dans ces conditions.

Les comportements analytiques de la mirtazapine et de la déméthylmirtazapine sont donc très dépendants des variations de $\mathrm{pH}$ dans des zones proches des deux $\mathrm{pKa}$.

\section{MOTS-CLÉS}

Mirtazapine, constante d'ionisation, CLHP, ECZ.

\section{Introduction}

La mirtazapine $(1,2,3,4,10,14 \mathrm{~b}$-hexahydro-2méthyl-pyrazino $[2,1$-a] pyrido [2,3-c] benzazépine), est un antidépresseur du groupe chimique des pipérazinoazépines. Elle est la première et la seule représentante de la classe des antidépresseurs noradrénergiques et sérotoninergiques ou NaSSA (Noradrenergic Specific Serotoninergic Antidepressants).

Elle peut être dosée dans les milieux biologiques par différentes méthodes chromatographiques (1-7). Maris et al (6) ont étudié la pharmacocinétique de la mirtazapine dans le plasma humain en chromatographie liquide haute performance (CLHP) avec une détection fluorimétrique. Ils sont les seuls à décrire une méthode permettant le dosage simultané de la mirtazapine et de son dérivé déméthylé actif. Anderson et al (1) et Moore et al (2) ont étudié la distribution tissulaire de la mirtazapine dans plusieurs cas médico-légaux par chromatographie en phase gazeuse (CG) couplée à un détecteur azote-phosphore ou à la spectrométrie de masse.

A côté de la classe des NaSSA, il existe deux autres classes de nouveaux antidépresseurs de type inhibiteur spécifique de la recapture de la sérotonine (ISRS) ou inhibiteur de la recapture de la sérotonine et de la noradrénaline (IRSNa) (8). De très nombreuses études décrivent l'identification et le dosage d'une ou plusieurs de ces molécules $(7,9$, revue générale : 10$)$. Les techniques les plus couramment utilisées sont la CLHP et la CG. Il n'existe actuellement pas de méthode de screening pour ces nouveaux antidépresseurs en électrophorèse capillaire de zone (ECZ) (11).

Faisant suite à des premiers travaux $(12,13)$, nous présentons dans cet article deux méthodes permettant la séparation de l'ensemble de ces nouveaux antidépresseurs. La première étude est réalisée en CLHP phase on a fused silica capillary with a migration buffer consisting of borate buffer $42 \mathrm{mM}$ (pH 9.5) and methanol (80:20, v/v), and an applied voltage of $15 \mathrm{kV}$.

In HPLC, we pointed out the major effect of the $\mathrm{pH}$ of the mobile phase ( $\mathrm{pH} 3$ to $\mathrm{pH} 4.95$ ) and of the temperature $\left(25^{\circ} \mathrm{C}\right.$ to $\left.50^{\circ} \mathrm{C}\right)$ on mirtazapine and demethylmirtazapine retention times. In CZE, the $p H$ of the migration buffer $(p H$ 9.2 to $\mathrm{pH}$ 9.9) has a similar and minor effect on all the antidepressant molecules migration, weakly ionized in these conditions.

In conclusion, the analytical behaviours of mirtazapine and demethylmirtazapine are very dependent of $p H$ changes in pH zones near the two pKas.

\section{KEY-WORDS}

Mirtazapine, ionisation constant, HPLC, CZE.

inverse sur une nouvelle phase stationnaire Satisfaction ${ }^{\circledR}$ RP 18 AB (Cluzeau Info Labo, France) couplée à un détecteur à barrette de diodes (12). La seconde étude est une première approche de la séparation de ces molécules en ECZ couplée à un détecteur à barrette de diodes (13). Lors de ces deux études, notre attention s'est portée plus particulièrement sur la mirtazapine et la déméthylmirtazapine. Ces deux molécules présentaient des comportements analytiques singuliers, différents de ceux des autres molécules antidépresseurs.

Ainsi, après avoir fait quelques rappels sur la pharmacologie et sur les propriétés acido-basiques de la mirtazapine, nous étudierons les comportements de cette molécule et de son métabolite actif dans ces deux méthodes de séparation pour différentes conditions opératoires.

\section{Rappels sur la pharmacolo- gie de la mirtazapine}

La mirtazapine est commercialisée en France depuis septembre 1999, sous le nom de Norset ${ }^{\circledR}$ (Organon, Eragny-sur-Epte, France) en boites de 30 comprimés pelliculés à $15 \mathrm{mg}$. Ses indicat: ons thérapeutiques sont les épisodes dépressifs majer', (14).

Elle est rapidement absorbée après administration orale avec une biodisponibilité de $50 \%$. Les concentrations plasmatiques sont dose-dépendantes avec un pic plasmatique ( $30 \mathrm{ng} / \mathrm{mL}-200 \mathrm{ng} / \mathrm{mL}$ ) atteint au bout de $2 \mathrm{~h}$ et une demi-vie plasmatique comprise généralement entre 20 et $40 \mathrm{~h}(8,15)$. Elle subit une déméthylation hépatique (cytochrome P450 3A), une hydroxylation (cytochrome P450 2D6 et 1A2) et une glucurocongugaison, à l'origine de nombreux métabolites dont la 
majorité est inactive. Seul le dérivé déméthylé, la déméthylmirtazapine, s'avère actif mais il est présent dans le plasma en concentration très faible. Approximativement $75 \%$ de la dose ingérée est éliminée dans les urines $(2,8,15)$.

Les études toxicologiques ne montrent pas d'effet cardiotoxique cliniquement significatif après surdosage en mirtazapine. Les symptômes de surdosage aigu sont limités à une sédation prolongée (8). Plusieurs cas mortels dans le cadre d'une polyintoxication pour laquelle la mirtazapine a été mise en évidence, ont été décrits dans la littérature. Les auteurs décrivent des concentrations de 30 à $570 \mathrm{ng} / \mathrm{mL}$ dans le sang cardiaque et de 40 à $240 \mathrm{ng} / \mathrm{mL}$ dans le sang fémoral périphérique (1-3).

\section{Matériel et méthodes} Réactifs

Tous les réactifs sont de qualité analytique. L'acétonitrile a été obtenu chez Baker (Deventer, Hollande), $\mathrm{KH}_{2} \mathrm{PO}_{4}, \mathrm{H}_{3} \mathrm{PO}_{4}$ et le méthanol chez Merck (Darmstadt, Allemagne), la triéthylamine chez Lancaster (Morecambe, UK) et le tétraborate de sodium $\left(\mathrm{Na}_{2} \mathrm{~B}_{4} \mathrm{O}_{7}, 10 \mathrm{H}_{2} \mathrm{O}\right)$ chez Riedel-de Haën (Seelze, Allemagne). La mirtazapine et la déméthylmirtazapine ont été gracieusement fournies par les laboratoires Organon (Eragny-sur-Epte, France), le citalopram $(\mathrm{HBr})$ et le déméthylcitalopram $(\mathrm{HBr})$ par Lundbeck A/S (Copenhague, Danemark), la fluvoxamine (maléate) par Solvay Pharma (Suresnes, France), le milnacipran $(\mathrm{HCl})$ et le standard interne $\mathrm{F} 2570$ par Pierre Fabre (Castres, France), la paroxétine $(\mathrm{HCl})$ par Smith Kline Beecham (Nanterre, France), la sertraline $(\mathrm{HCl})$ par Pfizer (Amboise, France) et la venlafaxine $(\mathrm{HCl})$ et la déméthylvenlafaxine par Lederle (Pearl River, N.Y., USA). La fluoxétine et la norfluoxétine ont été obtenues chez Sigma (Saint Quentin Fallavier, France).

Les solutions mères à $1 \mathrm{mg} / \mathrm{ml}$ de chaque molécule antidépresseur et de l'étalon interne sont préparées dans du méthanol et conservées à - $20^{\circ} \mathrm{C}$. Parallèlement, une solution mère contenant l'ensemble des molécules à des concentrations respectives de $1 \mathrm{mg} / \mathrm{ml}$ est préparée dans du méthanol. Les solutions de travail sont préparées extemporanément dans de la phase mobile en CLHP et dans de l'eau en ECZ, jusqu'à l'obtention d'une concentration finale de $20 \mu \mathrm{g} / \mathrm{ml}$ pour chìaque molécule. Toutes les solutions de travail sont filtrées à travers des filtres en nylon de $0,45 \mu \mathrm{m}$ avant leur injection en CLHP et en ECZ.

\section{Appareillage CLHP}

Les analyses sont réalisées sur une chaîne chromatographique constituée d'un injecteur manuel de type Rhéodyne modèle 7125 (boucle d'injection de $20 \mu \mathrm{l}$ ), d'une pompe SpectraSystem modèle P1000 (San Jose, CA, USA) et d'un détecteur à barrette de photodiodes Waters 990 (St Quentin en Yvelines, France). La séparation des 12 composés a été optimisée sur une colonne Satisfaction ${ }^{\circledR} \mathrm{RP} 18 \mathrm{AB}(250 \mathrm{~mm} \times 4,6 \mathrm{~mm}, 5 \mu \mathrm{m})$ (Cluzeau Info Labo, Sainte Foy La Grande, France) maintenue à une température de $45^{\circ} \mathrm{C}$ par un chauffe colonne de type CROCO-CIL ${ }^{\circledR}$ (Cluzeau Info Labo). La phase mobile est constituée par un mélange de tampon phosphate $25 \mathrm{mM}+$ triéthylamine $10 \mathrm{mM}(\mathrm{pH} 4,8)$ et d'acétonitrile $(65: 35, \mathrm{v} / \mathrm{v})$. Le débit est de $1 \mathrm{ml} / \mathrm{min}$ (12).

\section{ECZ}

Les analyses sont réalisées sur un appareil d'électrophorèse capillaire de type P/ACE MDQ (Beckman, Fullerton, CA, USA) avec une détection par barrette de photodiodes. La séparation a été optimisée sur un capillaire en silice fondue (1. totale $=60 \mathrm{~cm}, 1$. efficace $=50$ $\mathrm{cm}$, d.i. $=75 \mu \mathrm{m}$ ) maintenu à une température de $15^{\circ} \mathrm{C}$. Le tampon de migration est un mélange borate $(42 \mathrm{mM}, \mathrm{pH} 9,5)$ et méthanol $(80: 20, \mathrm{v} / \mathrm{v})$. On applique une tension de $15 \mathrm{kV}$ entre l'anode et la cathode pendant l'analyse. Les échantillons ont été injectés en mode d'injection hydrodynamique pendant 10 secondes à 0,5 psi (13).

Les conditions strictes de conditionnement du capillaire ont été décrites par ailleurs (16).

\section{Résultats et Discussion Propriétés acido-basiques de la mirtaza- pine}

Toutes les molécules de la classe des ISRS, des IRSNa et des NaSSA sont des molécules basiques comportant au moins une fonction amine. Ainsi, la fluvoxamine et le milnacipran possèdent une fonction amine primaire, la paroxétine, la fluoxétine et la sertraline une fonction amine secondaire, le citalopram et la venlafaxine une fonction amine tertiaire. La mirtazapine, quand à elle, possède deux fonctions basiques, une fonction amine, ici tertiaire et une deuxième fonction liée au groupement amino-2-pyridine (figure 1). La fonction amine tertiaire peut être déméthylée, se transformant ainsi en fonction amine secondaire dans la déméthylmirtazapine (figure 1). 


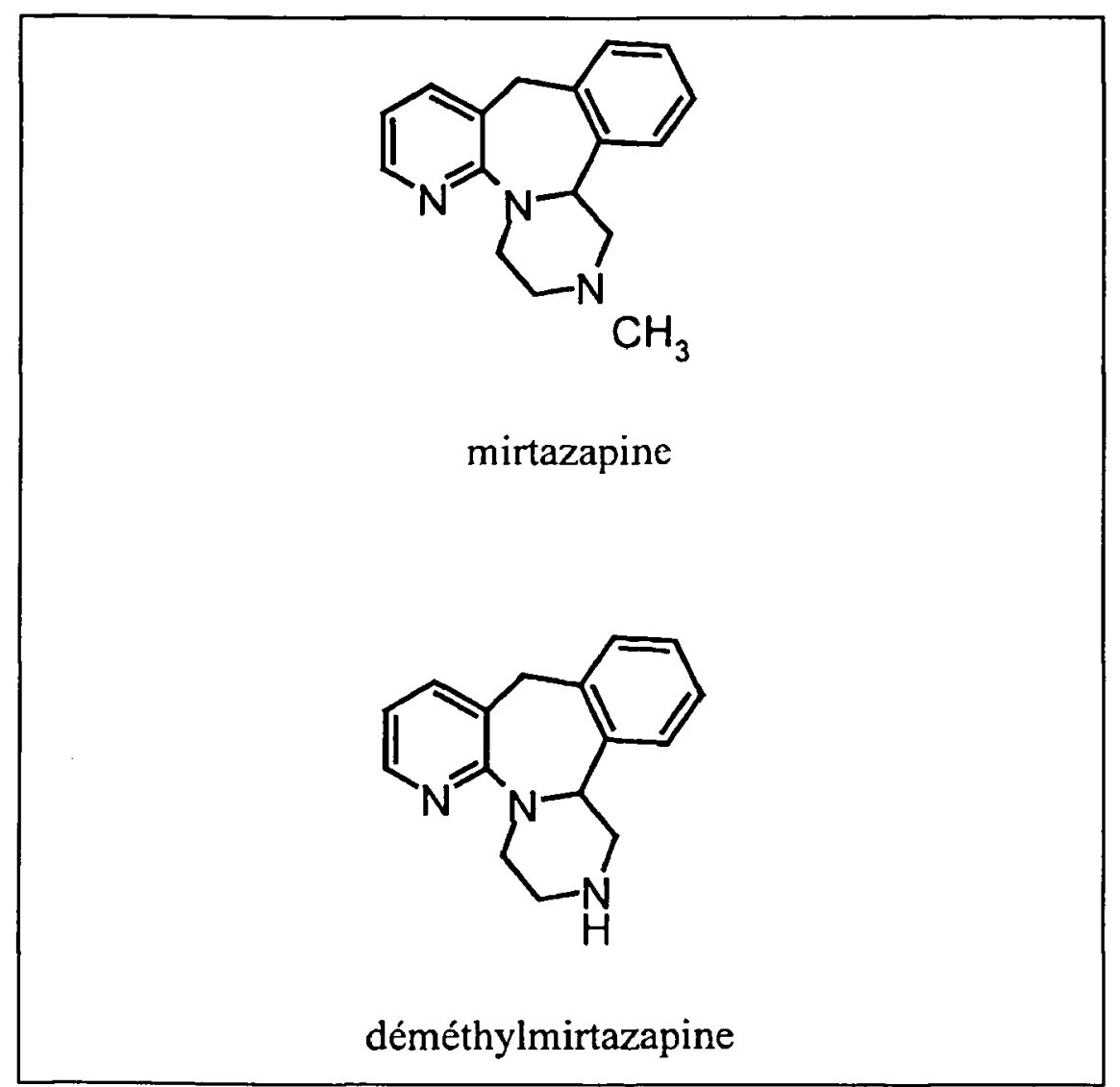

Figure 1 : Structures chimiques de la mirtazapine et de la déméthylmirtazapine.

Nous avons mesuré par potentiométrie ou spectrométrie UV les pKa de ces deux fonctions. Nous avons trouvé pour l'amine tertiaire une valeur de 7,50 $\pm 0,05$, similaire à celle obtenue par Kelder (17). Pour les molécules possédant une fonction amine primaire ou tertiaire, la valeur du pKa est généralement comprise entre 9 et 10 (18). Pour les molécules possédant une fonction amine secondaire la valeur est plus élevée, proche de 10,50. La valeur trouvée pour la mirtazapine est donc beaucoup plus faible.

Le deuxième $\mathrm{pKa}$ relatif au groupement amino-2-pyridine, a pour valeur 3,60 $\pm 0,05$ (résultats non publiés). Il est également beaucoup plus faible que celui classiquement décrit pour une fonction de type amino-2pyridine non substituée : pKa proche de 6,70.

Les valeurs faibles obtenues pour les deux pKa sont liées à la structure encombrée de la mirtazapine, rendant les doublets électroniques des azotes moins disponibles, à l'origine du caractère basique moins marqué de ces deux fonctions.

Face à ces caractéristiques acido-basiques tout à fait particulières, il nous a paru intéressant d'étudier le comportement analytique de la mirtazapine et de son métabolite parmi les autres antidépresseurs, dans le cadre du développement de méthodes de séparation en CLHP et en ECZ.

\section{Étude de la séparation des nouveaux antidépresseurs en CLHP}

Dans les conditions décrites plus haut, on obtient le chromatogramme représenté dans la figure 2. Ainsi, pour une durée d'analyse de l'ordre de 20 minutes, toutes les molécules sont parfaitement séparées avec une résolution et des facteurs de rétention satisfaisants ( $k$ compris entre 0,5 et 10). Dans ces conditions, la déméthylmirtazapine et la mirtazapine ont des temps de rétention (tR) respectifs de 3,63 et de 5,14 minutes (figure 2).

Lorsqu'on étudie l'influence du $\mathrm{pH}$ de la phase mobile sur la rétention des différentes molécules, on s'aperçoit de l'importance particulière de ce paramètre sur les tR de la mirtazapine et de son métabolite. En effet, lorsque le $\mathrm{pH}$ varie de 3 à 4,95 , on note un comportement singulier de la mirtazapine et à un moindre degré de la déméthylmirtazapine, avec une augmentation importante de leur rétention (figure 3). L'influence de la variation de $\mathrm{pH}$ sur la rétention de l'ensemble des dix autres molécules antidépresseurs est beaucoup moins marquée.

La figure 4 montre l'évolution des tR de cinq molécules (déméthylvenlafaxine, déméthylmirtazapine, milnacipran, mirtazapine et venlafaxine) lorsqu'on fait varier le pH de 3,50 à 4,95. Dans les chromatogrammes présentés, ces cinq antidépresseurs ont été choisis car leurs tR sont proches. Ainsi, lors de l'augmentation du $\mathrm{pH}$ de la phase mobile, les tR de la mirtazapine et de la déméthylmirtazapine augmentent nettement plus rapidement que celui des autres molécules. A pH 3,50, la mirtazapine est éluée en troisième position, alors qu'à $\mathrm{pH} 4,95$, elle est éluée la dernière, après les quatre autres molécules pour lesquelles l'ordre d'élution n'a pas changé.

Pour un $\mathrm{pH}$ de la phase mobile compris entre 3,50 et 4,95 , on se situe à proximité de la constante du premier équilibre acido-basique de la mirtazapine $(\mathrm{pKa}=3,6)$. Il paraît alors tout à fait normal qu'une variation de $\mathrm{pH}$ dans cette zone ait une grande influence sur les conditions d'ionisation de cette molécule et donc sur son temps de rétention en CLHP. La déméthylmirtazapine qui possède une structure voisine, présente un comportement similaire. La variation de son tR en fonction du pH est cependant moins nette, car cette molécule, plus polaire que la mirtazapine, est éluée près du temps mort dans les conditions décrites. Pour les autres molécules antidépresseurs, l'existence d'une seule constante acido-basique proche de 9 ou de 10 explique leur comportement homogène et moins influencé par le $\mathrm{pH}$ dans une zone acide variant entre $\mathrm{pH} 3$ et $\mathrm{pH} 5$.

L'influence de la température d'analyse sur la séparation de ces molécules en CLHP a également été étu- 


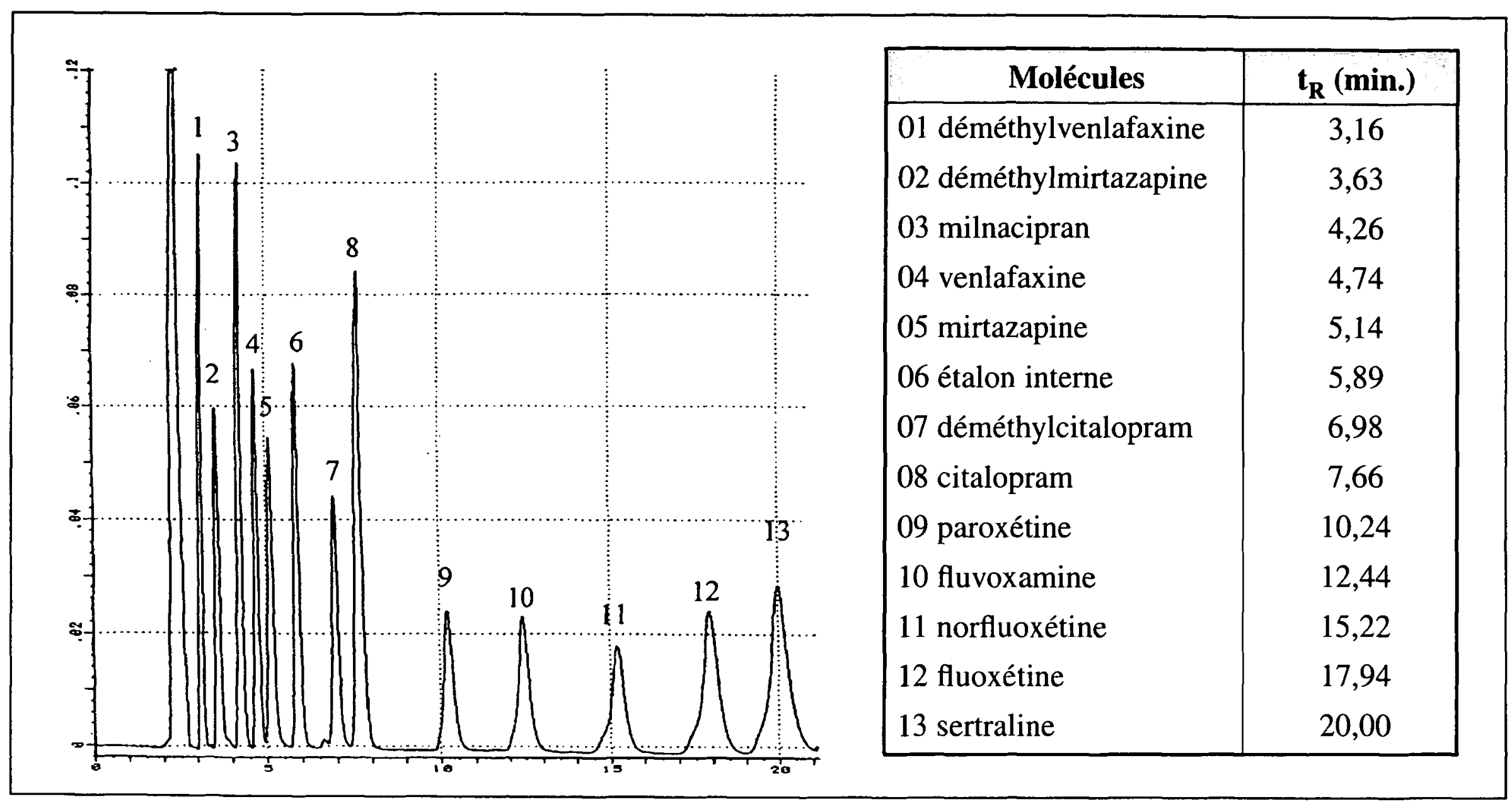

Figure 2 : Chromatogramme d'un mélange des douze antidépresseurs réalisé à $45^{\circ} \mathrm{C}$ pour une phase mobile de composition : acétonitrile $\mathrm{KH}_{2} \mathrm{PO}_{4}(25 \mathrm{mM})$ et triéthylamine $(10 \mathrm{mM}) \mathrm{pH} 4,8(35: 65, \mathrm{v} / \mathrm{v})$.

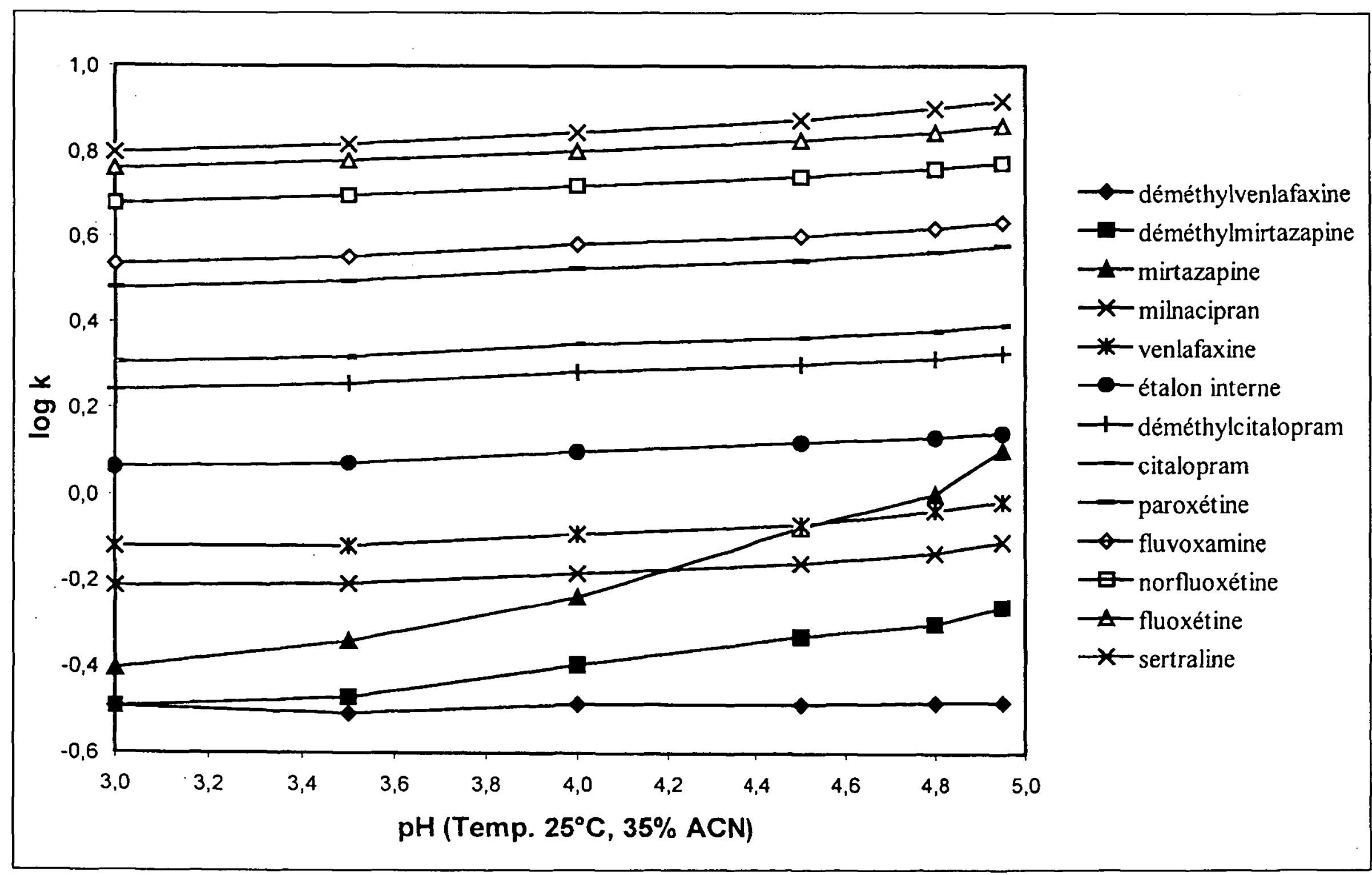

Figure 3 : Influence du pH de la phase mobile en CLHP sur les facteurs de rétention des douze antidépresseurs. 

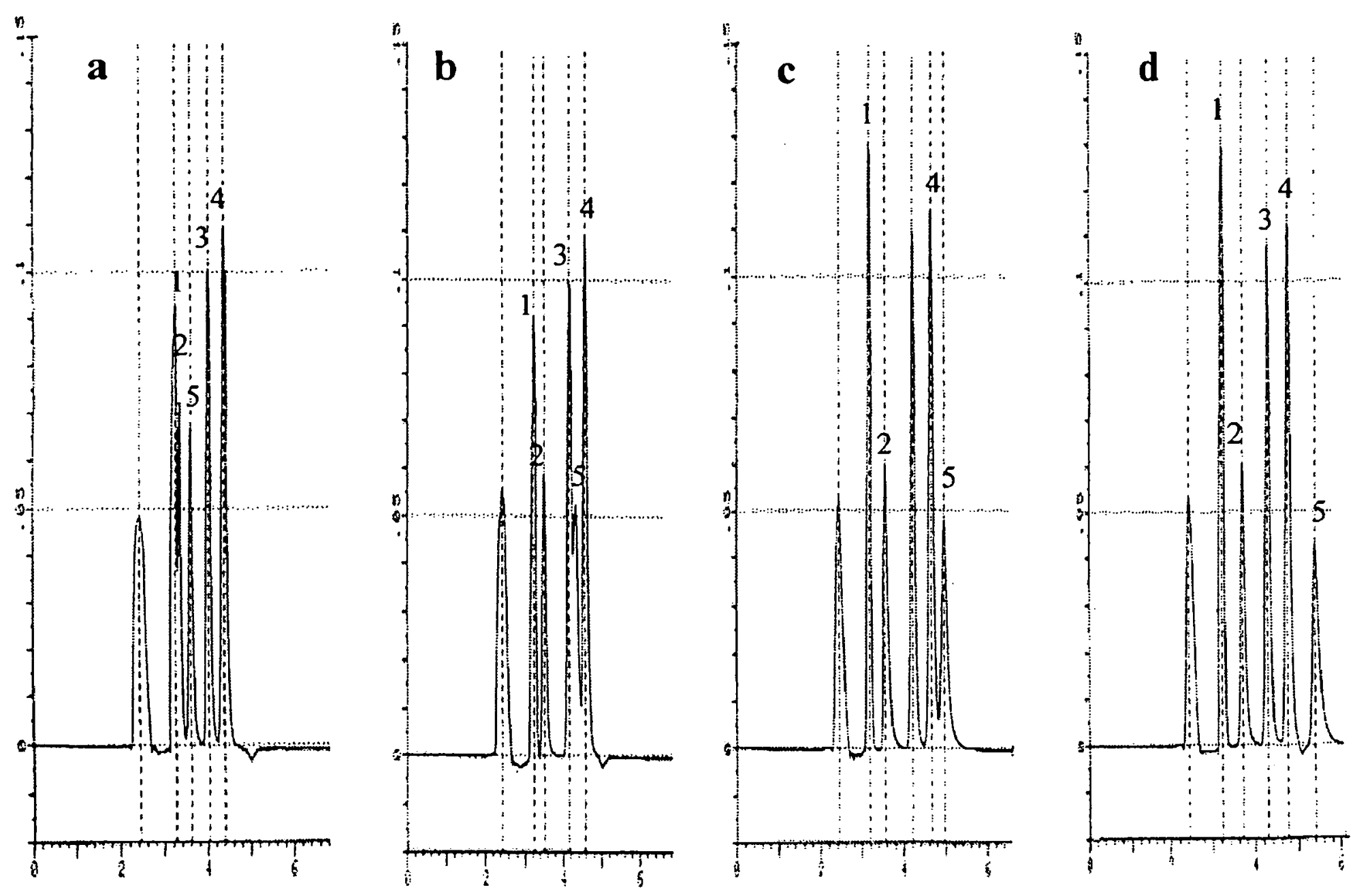

Figure 4 : Chromatogrammes d'un mélange de cinq antidépresseurs (1/ déméthylvenlafaxine, 2/ déméthylmirtazapine, 3/ milnacipran, 4/venlafaxine et $5 /$ mirtazapine) obtenus à $25^{\circ} \mathrm{C}$ pour différents $p H$ de la phase mobile $: a / p H 3,5 ; b / p H 4,5 ;$ $c / p H 4,8 ; d / p H 4,95$.

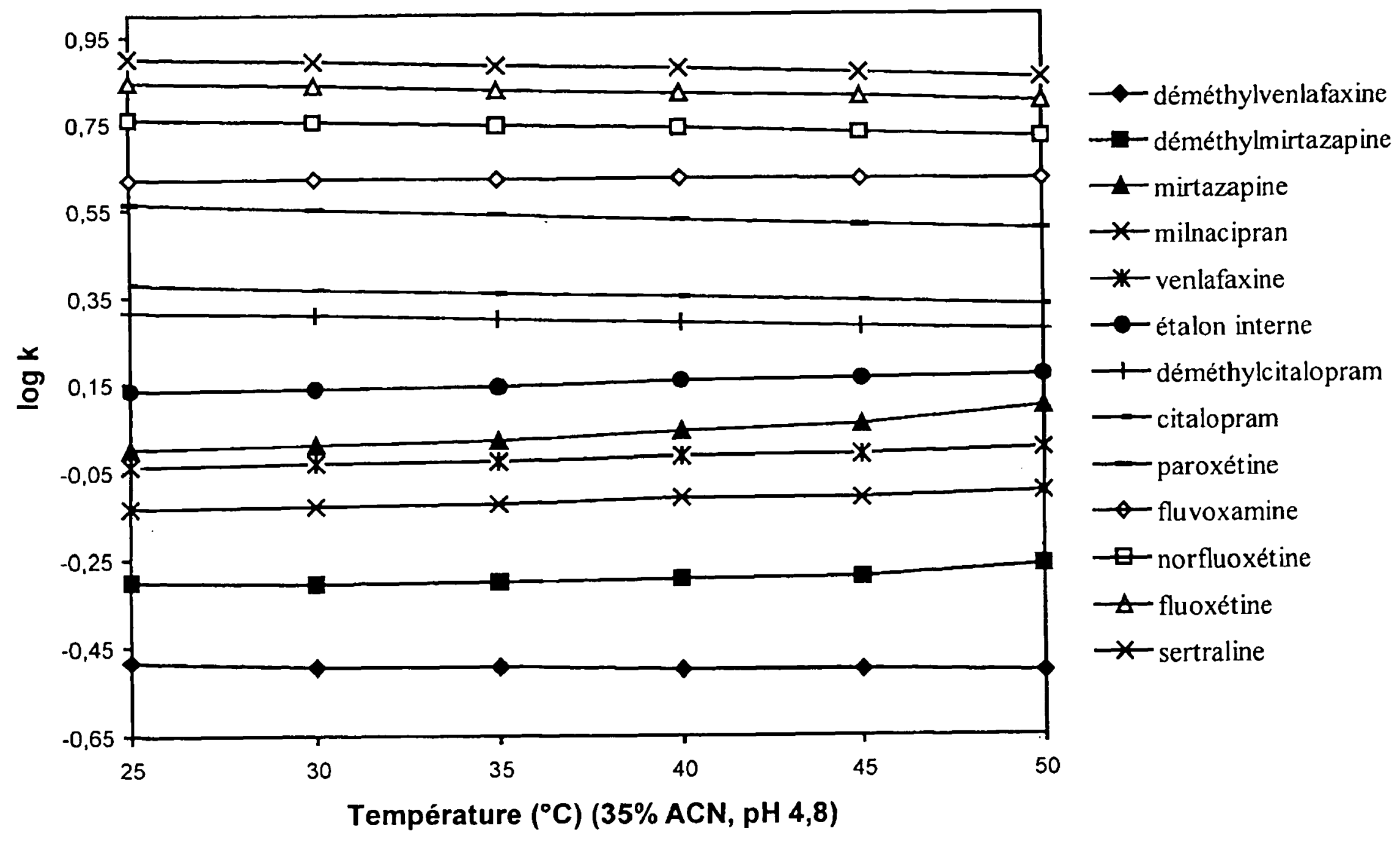

Figure 5 : Influence de la température d'analyse en CLHP sur les facteurs de rétention des douze antidépresseurs. 
diée. On retrouve lors de cette étude, un comportement particulier de la mirtazapine et de la déméthylmirtazapine (figure 5). Alors que l'on observe une légère diminution des tr de l'ensemble des molécules lorsque la température d'analyse varie de $25^{\circ} \mathrm{C}$ à $50^{\circ} \mathrm{C}$, la rétention de la mirtazapine et de son métabolite augmente. Cette constatation peut s'expliquer par l'influence de l'équilibre acido-basique liée à la fonction amino-2pyridine de la mirtazapine dans la zone de $\mathrm{pH}$ choisie. En effet, on se retrouve à proximité d'un équilibre thermodynamique, donc dépendant entre autre de la température.

Il est donc primordial de réaliser ce type de screening en CLHP dans des conditions de $\mathrm{pH}$ et de température parfaitement définies. Nous avons montré qu'une très faible variation de l'un de ces deux paramètres peut être à l'origine d'une modification assez importante des tR de la mirtazapine et de la déméthylmirtazapine, et donc de coélutions avec les autres antidépresseurs.

\section{Étude de la séparation des nouveaux antidépresseurs en $\mathbf{E C Z}$}

Dans les conditions opératoires décrites dans le paragraphe matériel et méthodes, la séparation des onze molécules antidépresseurs est possible en ECZ en moins de 18 minutes. Un exemple d'électrophérogram- me est donné dans la figure 6 . On peut observer la séparation de la majorité des molécules entre la $12^{\text {ieme }}$ et la $16^{\mathrm{i} m e}$ minute alors que la mirtazapine et la déméthylmirtazapine ont des temps de migration (tM) plus importants, respectivement de 17,06 et 17,54 minutes. Ces deux molécules migrent à proximité du flux électroosmotique (FEO), qui correspond à la migration des molécules non chargées (16). Ces tM plus importants peuvent s'expliquer simplement par une différence de l'état d'ionisation de la mirtazapine et de son métabolite dans les conditions opératoires choisies. $\mathrm{Au} \mathrm{pH}$ du tampon de migration ( $\mathrm{pH} 9,5$ ), ces molécules se retrouvent dans un état d'ionisation très faible (pKa mesuré pour la mirtazapine à 7,5 ) expliquant ainsi leurs migrations proches du FEO et leur excellente séparation d'avec les autres antidépresseurs. A ce $\mathrm{pH}$, ces derniers se retrouvent dans un état d'ionisation comparable, ont des mobilités électrophorétiques voisines et migrent plus rapidement que les molécules neutres (marquées par le FEO). Seule la présence d'un modificateur organique comme le méthanol additionné au tampon de migration permet de les séparer en ECZ.

Comme en CLHP, nous avons étudié l'influence de plusieurs paramètres comme le $\mathrm{pH}$ ou le pourcentage en méthanol dans le tampon de migration, la température d'analyse et la tension appliquée entre l'anode et la cathode sur les tm des différentes molécules. La varia-

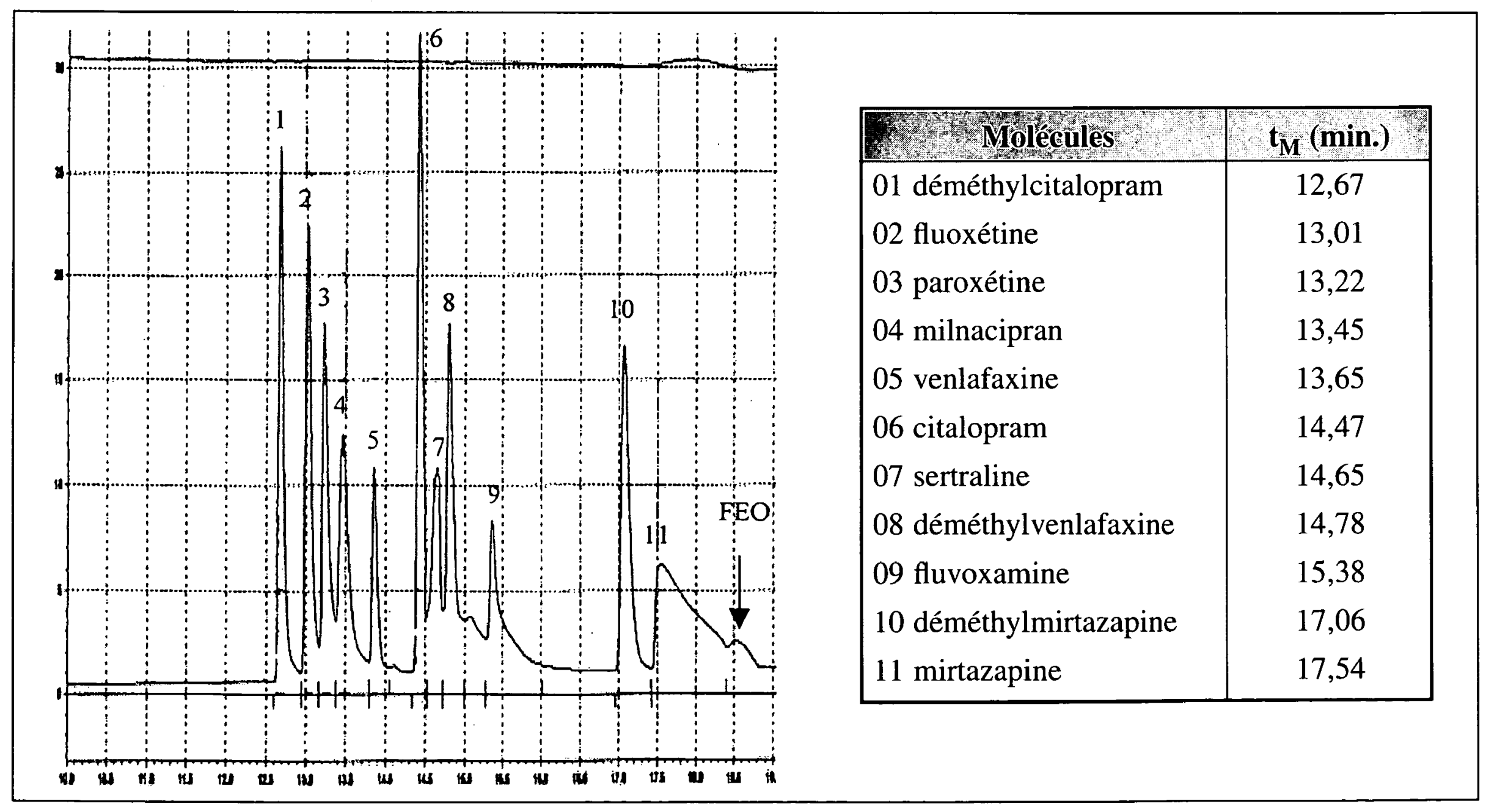

Figure 6 : Electrophérogramme d'un mélange de onze antidépresseurs, réalisé à $15^{\circ} \mathrm{C}$ pour une tension appliquée de $15 \mathrm{kV}$ et un tampon de migration de composition: méthanol/borate $42 \mathrm{mM} \mathrm{pH} \mathrm{9,5} \mathrm{(20:80,v/v).}$ 
tion du $\mathrm{pH}$ du tampon de migration, entre 9,2 et 9,9, a une influence homogène sur tous les antidépresseurs y compris sur la mirtazapine et la déméthylmirtazapine : on observe une augmentation de l'ensemble des $\mathrm{tM}$ pour les $\mathrm{pH}$ les plus basiques. Il n'a pas été possible d'améliorer la séparation de la mirtazapine et du FEO. Une diminution plus nette du $\mathrm{pH}$ serait nécessaire pour cette séparation, permettant d'augmenter l'état d'ionisation de la mirtazapine et de son métabolite, mais rendant alors la séparation des autres molécules impossible en ECZ (19). De la même façon, l'augmentation du pourcentage en méthanol de 0 à $20 \%$ dans le tampon de migration, la diminution de la température d'analyse de 40 à $15^{\circ} \mathrm{C}$ ou la diminution de la tension appliquée pendant l'analyse de 30 à $12 \mathrm{kV}$ entraînent une augmentation des tM de l'ensemble des molécules avec des valeurs restant plus élevées pour la mirtazapine et la déméthylmirtazapine (résultats non présentés).

Lors de cette optimisation en ECZ, le pH est basique et très différent de celui que nous avions choisi en CLHP. On est ainsi plus éloigné du deuxième équilibre acidobasique de la mirtazapine ( $\mathrm{pKa}=7,5$ ). Le choix de cette zone de $\mathrm{pH}$ a pour conséquence une faible variation de l'état d'ionisation de la mirtazapine et de la déméthylmirtazapine qui sont déjà peu ionisées, lorsqu'on fait varier le $\mathrm{pH}$ du tampon de migration de 9,2 à 9,9. Les autres antidépresseurs dont le pKa varie de 9 à 10,5 , se retrouvent ionisés sous forme de cations, et migrent ainsi plus rapidement que le FEO. Ces composés subissent peu l'influence d'une variation de $\mathrm{pH}$ dans la fourchette choisie.

\section{Conclusion}

Les comportements analytiques de la mirtazapine et de la déméthylmirtazapine sont très dépendants des variations de $\mathrm{pH}$ dans des zones proches des deux $\mathrm{pKa}(3,6$ et 7,5). Ainsi, en CLHP on observe entre $\mathrm{pH} 3$ et $\mathrm{pH} 5$ un changement de l'état d'ionisation de la mirtazapine à l'origine d'une variation importante de son tR. La majorité des études décrites dans la littérature pour le dosage des nouveaux antidépresseurs est réalisée à un $\mathrm{pH}$ acide en général proche de $\mathrm{pH} 4(9,20)$. Dans ces conditions, il est donc important de préparer les phases mobiles dans des conditions parfaitement définies, permettant d'obtenir un $\mathrm{pH}$ précis. Les deux molécules sont beaucoup moins sensibles aux variations de $\mathrm{pH}$ dans des zones plus basiques utilisées en ECZ.

Les résultats des deux techniques présentées sont une illustration du fait qu'il n'existe pas de similitude entre un screening réalisé en CLHP et en ECZ (21). La séparation présentée en CLHP est plus performante que celle obtenue en ECZ. Ceci peut s'expliquer par le choix des conditions opératoires présentées en ECZ dans lesquelles les molécules comme la mirtazapine se retrouvent dans un état d'ionisation très faible.

Les caractéristiques de migration des molécules d'une même famille chimique deviennent différentes en présence de micelles et sont moins dépendantes de la valeur du pKa $(16,21)$. Ainsi, de nouvelles études utilisant les performances de l'électrophorèse capillaire en mode micellaire sont en cours de réalisation pour permettre d'améliorer la séparation des nouveaux antidépresseurs.

\section{Références}

1. Anderson D.T., Fritz K.L., Muto J.J., Distribution of mirtazapine (Remeron $®$ ) in thirteen postmortem cases. $J$. Anal. Toxicol. $1999 ; 23: 544-8$.

2. Moore K.A., Levine B., Smith M.L., Saki S., Schames J., Smialek J.E., Tissue distribution of mirtazapine (Remeron ${ }^{\circledR}$ ) in postmortem cases. J. Anal. Toxicol. $1999 ; 23: 541-3$.

3. Lavins E.S., Wogoman H.M., Jenkins A.J., Mirtazapine (Remeron $($ ) : detection of a new antidepressant in postmortem cases. TIAFT-SOFT joint congress, 4 octobre 1998, Albuquerque (US).

4. Paanakker J.E., van Hal H.J., Capillary gas chromatographic assay for the routine monitoring of the antidepressant mepirzepine in human plasma. J. Chromatogr. $1987 ; 417$ (1) : 203-7.

5. Dodd S., Burrows G.D., Norman T.R., Chiral determination of mirtazapine in human blood plasma by high-performance liquid chromatography. J. Chromatogr B $2000 ; 748: 439-43$.
6. Maris F.A., Dingler E., Niehues S., High-performance liquid chromatographic assay with fluorescence detection for the routine monitoring of the antidepressant and its demethyl metabolite in human plasma. J. Chromatogr. B Biomed. Sci. Appl. 1999 ; 721 (2) : 309-16.

7. Eap C.B., Baumann P., Analytical methods for the quantitative determination of selective serotonin reuptake inhibitors for therapeutic drug monitoring purposes in patients. J. Chromatogr. B Biomed. Appl. 1996 ; 686 (1) : 51-63.

8. Kent J.M., SnaRIs, NaSSAs, and NaRIs : new agents for the treatment of depression. Lancet $2000 ; 355$ (9207) : 911-8.

9. Lacassie E., Gaulier J.M., Marquet P., Rabatel J.F., Lachatre G., Methods for the determination of seven selective serotonin reuptake inhibitors and three active metabolites in human serum using high-performance liquid chromatography and gas chromatography. J. Chromatogr. B Biomed. Sci. Appl. 2000 ; 742 (2) : 22938. 
10. Maurer H.H., Bickeboeller-Friedrich J., Screening procedure for detection of antidepressants of the selective serotonin reuptake inhibitor type and their metabolites in urine as part of a modified systematic toxicological analysis procedure using gas chromatography-mass spectrometry. J. Anal. Toxicol. 2000 ; 24 (5) : 340-7.

11. Labat L., Lhermitte M. Les nouvelles technologies au service de la toxicologie clinique. Ann. Toxicol. Anal. 2000 ; XII (4) : 340-51.

12. Dallet P., Labat L., Kummer E., Dubost J.P., Séparation des antidépresseurs de nouvelle génération par CLHP sur colonnes Satisfaction ${ }^{\circledR}$ RP 18 AB et Stability ${ }^{\circledR}$ C8. Eur. J. Emerg. Med. $2001 ; 8$ : 67-82.

13. Labat L, Deveaux M, Dumestre-Toulet V., Dallet P., Kummer E.; Dubost J.P., Intoxications par les nouveaux antidépresseurs : screening en électrophorèse capillaire de zone. Eur. J. Emerg. Med. 2001 ; 8 : 67-82.

14. Gorman J.M., Mirtazapine : Clinical overview. J. Clin. Psychiatry $1999 ; 60$ (17) : 9-13.

15. Timmer C.J., Sitsen J.M., Delbressine L., Clinical pharmacokinetics of mirtazapine. Clin. Pharmacokinet. $2000 ; 38(6): 461-74$.

16. Labat L., Deveaux M., Dubost J.P., Applications de l'électrophorèse capillaire en toxicologie clinique et médico-légale. Ann. Toxicol. Anal. 2000 ; XII (3) : 179195.
17. Kelder J., Funke C., De Boer T., Delbressine L., Leysen D., Nickolson V., A comparison of the physicochemical and biological properties of mirtazapine and mianserine. J. Pharm. Pharmacol. 1997 ; 49 (4) : 403-11.

18. Rudaz S., Stella C., Balant-Gorgia A.E., Fanali S., Veuthey J.L., Simultaneous stereoselective analysis of venlafaxine and O-desmethylvenlafaxine enantiomers in clinical samples by capillary electrophoresis using charged cyclodextrins. J. Pharm. Biomed. Anal. 2000 ; 23 : 107-15.

19. Buzinkalova T., Polonaky J., Determination of four selective serotonin reuptake inhibitors by capillary isotachophoresis. Electrophoresis $2000 ; 21: 2839-41$.

20. Kristoffersen L., Bugge A., Lundanes E., Slordal L., Simultaneous determination of citalopram, fluoxetine, paroxetine and their metabolites in plasma and whole blood by high-performance liquid chromatography with ultraviolet and fluorescence detection. J. Chromatogr. B 1999 ; $734: 229-46$.

21. Boone C.M., Franke J.P., de Zeeuw R.A., Ensing K., Evaluation of capillary electrophoretic techniques towards systematic toxicological analysis. J. Chromatogr. A $1999 ; 838(1-2): 259-72$. 